\title{
Los relatos de vida como técnica para abordar la dimensión estructural del mundo social
}

\section{Resumen:}

En este artículo se reflexiona sobre los relatos de vida para conocer condiciones estructurales, posiciones de clase social y mecanismos objetivos de funcionamiento de ámbitos específicos de actividad humana. En la primera parte se retoman aportes de Daniel Bertaux sobre las características de los relatos de vida y sobre la técnica de graficar trayectorias laborales y educativas familiares en árboles genealógicos. Luego, a partir de un ejemplo de investigación empírica sobre trayectorias de clase en Buenos Aires, se destacan las posibilidades que exhibió la aplicación de estas técnicas y algunos atributos adicionales a los que habían sido señalados por Bertaux.

Palabras clave: métodos biográficos, relatos de vida; árboles genealógicos; etnosociología; familias

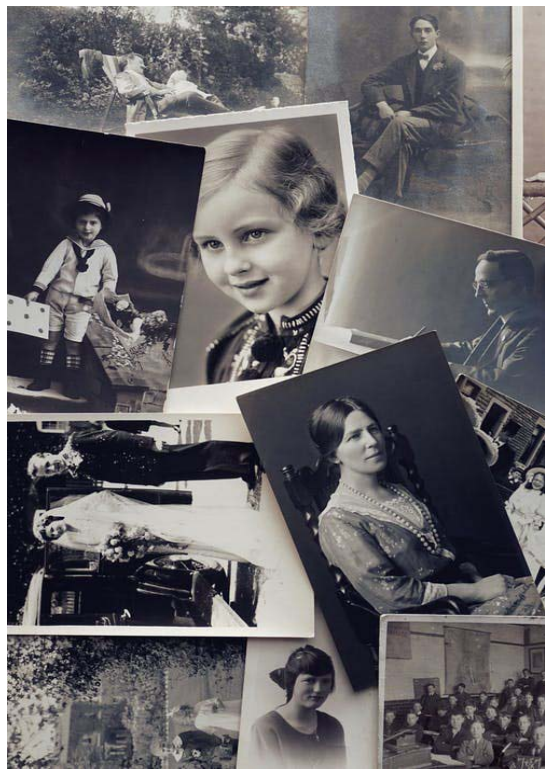
and some additional attributes to those that had been pointed out by Bertaux.

Keywords: biographical methods, life stories; family trees; ethnosociology; familie

Gonzalo Seid: gonzaloseid@gmail.com Doctor en Ciencias Sociales (Universidad de Buenos Aires). Becario posdoctoral del Consejo Nacional de Investigaciones Científicas y Técnicas. Temas de investigación: clases sociales, desigualdades; metodología de la investigación. ORCID: https://orcid. org/0000-0002-1242-9301Recibido, 31/07 /20, Publicado05/10/20 
las propuestas teórico-metodológicas de Bertaux, sobre trayectorias y mecanismos de movilidad social de familias en Buenos Aires en las últimas décadas. La experiencia de esta investigación muestra alguno elementos sobre la utilidad de la técnica, que se integran con las que había señalado Bertaux. de investigación social es habitual que se contrapongan las características generales $\mathrm{d}$ los abordajes cuantitativos y cualitativos, pero también es extendido el cuestionamiento a la idea de confrontación entre paradigmas metodológicos. Actualmente hay consenso en la necesidad de pluralismo metodológico, en que los métodos se complementan y en que la elección de métodos y técnicas depende de preguntas, hipótesis y objetivos de investigación. En el presente artículo nos proponemos argumentar cómo el método biográfico mediante relatos de vida puede servir a objetivos que exceden la comprensión de significados, discursos y puntos de vista subjetivos, que normalmente se atribuyen a las técnicas cualitativas basadas en entrevista.

Para mostrar otras posibilidades de uso de los relatos de vida, se

retoma la propuesta de Daniel Bertaux sobre la estrategia etnosociológica, que apunta a describir aspectos estructurales de la realidad social mediante metodologías que tradicionalmente se asociaron a otro tipo de objetivos. Además, se expone la complementariedad entre relatos de vida y árboles genealógicos como técnicas de investigación. Luego, se presentan alguna consideraciones metodológicas surgidas de una investigación empírica ${ }^{1}$, inspirada en

1Se trata de la investigación para mi tesis doctoral Trayectorias de clase y género a través de relatos de vida: la intersección de desigualdades, dirigida por Gabriela Gómez Rojas, quien me dio a conocer los trabajos de Daniel Bertaux.

de documentos tales como autobiografías, biografías, diarios, cartas, notas necrológicas o crónicas de experiencias personales. Progresivamente fueron aceptados también los documentos no textuales, como fotografías y objetos materiales. En la actualidad, los documentos de los nativos incluyen todo tipo de registros textuales y audiovisuales en plataformas virtuales, redes sociales, blogs, etcétera.

Además de los documentos - en el sentido amplio que incluye lo textual, lo audiovisual y lo material— que se producen permanentemente para distintos fines en la vida cotidiana, los métodos biográficos suponen la construcción de materiales ex un fragmento de la historia de una vida tal profeso para la investigación, por ejemplo, los relatos de vida producidos en entrevistas biográficas. Daniel Bertaux ha sugerido una definición minimalista de relato de vida: este existe desde el momento en que una persona cuenta a otra un episodio cualquiera de su experiencia de vida.

En la investigación social habitualmente se distinguen - y confunden - los relatos de vida y las historias de vida. La denominación relato de vida - lifestory — designa como la cuenta quien la ha vivido. En cambio, historia de vida - life history - refiere a una reconstrucción biográfica realizada a partir de un estudio de caso sobre un actor dado, recurriendo a cualquier tipo de información y documentos, entre ellos su propio relato.

Las historias de vida suponen un abordaje de la vida entera de una persona, en todas sus dimensiones, profundizando en los más variados aspectos y procurando describir de manera exhaustiva su biografía completa. Desde la sociología, Bertaux sostiene que

[...] hay que liberarse de la poderosa influencia del modelo autobiográfico. Aqu no se trata de intentar comprender a un individuo determinado, sino una partede un objeto social. (Bertaux, 2005, p. 49)
Cuando una investigación pretende teorizar a partir de los datos - y no solo describir casos-, pueden ser preferibles los relatos de vida, porque prevén recortes temporales y temáticos, una mayor gravitación de los propósitos teóricos y una menor relevancia de la biografía en sí misma - en comparación a las historias de vida o a una biografía estándar- En la investigación social un relato de vida es :

[...]una improvisación sin notas (sin el recurso a los archivos escritos), que se basa en la rememoración de los principales acontecimientos tal como fueron vividos, memorizados y totalizados, poniendo sumo cuidado en discernir su concatenación. (Bertaux, 2005, p. 78)

El relato de vida es un relato de prácticas, de la acción en situación, que toma a los entrevistados como informantes sobre los contextos y las estructuras de oportunidades locales que conforman su vida, sus experiencias, acciones y percepciones.

En este sentido, Bertaux ha propuesto un estilo de investigación cualitativa, la etnosociología, que tiene como objetivo estudiar un fragmento particular de la realidad socio-histórica, priorizando las relaciones y procesos sociales estructurales. La investigación etnosociológica se basa en el trabajo de campo, inspirado en la tradición etnográfica de observación de lo particular pero sus problemáticas son sociológicas y tienen una pretensión de generalidad. El uso que Bertaux propone para los relatos de vida en la investigación social está enmarcado en esta perspectiva etnosociológica.

En lo epistemológico, la perspectiva etnosociológica reivindica el postulado realista según el cual la historia de un sujeto posee una realidad previa e independiente de su relato. Adiferencia de las concepciones "textualistas", que se interesan exclusivamente por el nivel semántico, la etnosociología reivindica que los relatos de vida también pueden usarse para conocer la dimensión la histo recorrido integrador a través de la "to el ideal de la autobiografía, se requiere un esfuerzo podría llegar a tener un gran expresivo, como retrato vivo de realidac históricas de interés, pero resulta cuestion su valor intrínseco para la producción de conocimiento científico, por no delimita desde la teoría su objeto de estudio y po tratarse de la historia de una sola persona o de unas pocas. dad" de la experiencia de un actor,

.

Revista Perspectivas Metodológicas | Universidad Nacional de Lanús | ISSN 2618-4125| 
objetiva o estructural de la vida social. Si bien algunos podrían considerar obvia esta posibilidad, lo "novedoso" de esta postura se debe a que en los textos y discursos metodológicos sobre investigación cualitativa el énfasis suele ponerse en la comprensión de significados subjetivos.

Aquel énfasis recurrente en que las investigaciones cualitativas en general, y la técnica de entrevista en especial, permiten aprehender lo particular, lo vivido subjetivamente y el plano del discurso, ha hecho perder de vista que también pueden ser utilizadas legítimamente para producir información sobre condiciones objetivas y procesos estructurales, así como para inferi mecanismos de funcionamiento que contribuyen a explicar los procesos y las relaciones en distintos ámbitos de actividad humana.

Lo que suele tomarse como un carácter constitutivo de los relatos de vida — a saber, que su valor particular reside en su capacidad de comprender «desde el interior» los procesos anómalos- no es más que una de sus múltiples facetas puestas de reliev por una escuela particular, la escuela de Chicago. (Bertaux, 1999, p. 5)

Los abordajes cuantitativos - típicament mediante encuesta - no tienen por que tener exclusividad para el relevamiento de información sobre condiciones objetivas. No es legítimo considerarlos más objetivos o menos sesgados, ni siquiera más fiables. Los datos de encuesta provienen de respuestas que no dejan de ser subjetivas y que luego son codificadas según decisiones teóricometodológicas. Si se confía en el respondente de una encuesta:

[...] ¿ por qué no habría de creérsele cuando ofrece esa misma información en el marco de una entrevista prolongada cara a cara, donde es mucho más difícil mentir? (Bertaux, 2005, p. 24)

Las informaciones fácticas proporcionadas por los sujetos en sus relatos de vida resultan por lo menos igual de exactas y fia- bles que las recogidas mediante cuestionario estandarizado, pero a menudo contienen mayor riqueza, porque son informaciones que incluyen los contextos de distinto tipo -social, macro-histórico, de roles y biográfico-que enmarcan acciones, sucesos $\mathrm{y}$ transiciones.

En la entrevista el relato de vida del sujeto se produce en diálogo con el investigador quien lo orienta para que rememore sus experiencias pasadas a través de un filtro teóricamente definido. Ello contribuirá a centrar la evocación en las dimensiones de biografía más vinculadas a los procesos y los mecanismos sociales de interés. Los relatos de vida son casos particulares, pero pueden ser puestos en relación con otros relatos integrados en contextos generales, con el fin de descubrir regularidades y patrones. Los relatos de vida son discursos, pero discursos que narran historias reales - de un plano de lo real que precede a los discursosSon producidos intersubjetivamente en la relación dialógica de entrevista, pero las mediaciones subjetivas y culturales a través de las cuales se narra la experiencia vivida no obligan a que el análisis permanezca en ese plano.

La interpretación subjetiva de significados del discurso es condición de posibilidad del relato de vida, pero no implica que el objeto de estudio deba circunscribirse al plano de análisis de la realidad discursiva de relato. Bertaux cuestiona las concepciones "antirrealistas", para las que no tiene sentido hablar de historia "realmente vivida" o consideran incierta la relación entre relato e historia. Siempre hay:

[...]buena parte de selección y de interpretación, sin la cual no habría más que una sucesión de hechos, un curriculum vitae sin articulaciones, ni se puede negar, ni se puede evitar: de otro modo no habría relato. (Bertaux, 2005, p. 79)

Si bien no se puede pretender reconstrui objetivamente y por completo un itinerario biográfico, el relato no puede tratarse como una mera ficción.

Los casos particulares importan en tanto manifestación de los fenómenos sociales. Para poner el relato de vida al servicio de la investigación etnosociológica se necesita trabajar con varios relatos. Disponer de un conjunto de testimonios permite aprehender el núcleo común a las distintas experiencias. Así, se abstrae lo que estas tienen de colectivo, los procesos y mecanismo sociales subyacentes. Además, enfocar el análisis en lo que hay de colectivo en el relato permite eludir "[...] esa parte de coloración retrospectiva que pueda haber" (Bertaux 2005: 41), obstáculo que Pierre Bourdieu conceptualizó como ilusión biográfica. Este modo de concebir los relatos supone explícitamente conectar las voces de los sujetos con los factores objetivos:

[...] ] través de la narración vemos trayectorias que nos señalan cual es el movimiento de los sujetos entre los mecanismos que conectan relaciones, objetos y sujetos en el proceso continuo de constitución social. (Scribano, 2008, p. 104)

Las lógicas que rigen distintos "mundos sociales" pueden captarse observando algunos microcosmos. Si se toma a los entrevistados como informantes que, por pertenecer a determinado campo de actividad o categoría de situación, poseen ciertos saberes sobre su funcionamiento interno - saberes que desconocen quienes no han pasado por esas experiencias-, es posible confiar en que sus relatos permitan comprender prácticas recurrentes y así estudiar procesos estructurales.

Los relatos de vida permiten un abordaje privilegiado del carácter diacrónico de la realidad social y de las vidas de los agentes. La dimensión temporal del recorrido vivencial es una clave para captar "la lógica de la acción en su desarrollo biográfico, y la configuración de las relaciones sociales en su desarrollo histórico (reproducción y dinámica de transformación)" (Bertaux,
2005: 11). El trabajo con los relatos conlleva: seleccionar hechos narrados, interpretarlos, contextualizarlos, identificar las concatenaciones con otros hechos, reconstruir la cronología, y reorganizar la historia. Establecer el orden temporal de los fenómenos es una condición previa y necesaria para poder conjeturar mecanismos sociales.

Uno de los ejes centrales de este tipo de análisis consiste en buscar los índices y los turningpoints - puntos de inflexión(Kornblit, 2007). Los índices son aquellos aspectos que los entrevistados o los investigadores reconocen como hechos que han marcado la experiencia de vida. Los puntos de inflexión o momentos bisagra son aquellos en que se presentó una encrucijada a partir de la cual ocurrió un viraje, un cambio en el itinerario biográfico con respecto al rumbo previo. Resulta de interés indagar cómo se llegó a tal punto y qué llevo al agente a elegir un rumbo en vez de otro en un momento determinado. Los virajes o incidentes críticos pueden ser de distinto tipo: un evento principal que alcanza todas las dimensiones de la vida de la persona (ej. migración), un evento que es producto de una acumulación de experiencias (ej. divorcio luego de conflictos de pareja) o un evento en apariencia poco importante en $s$ mismo pero que representa simbólicamente la transformación (ej. "epifanía”, toma de conciencia o cambio radical en la forma de ver algo significativo). Los virajes articulan factores objetivos con las percepciones de los sujetos y pueden implicar diversos grados de ruptura con la identidad previa.

Para que sea posible acceder a procesos mecanismos estructurales, es necesario distinguir en los relatos tres órdenes de realidad diferentes:

— La realidad discursiva del relato en su producción dialógica en la situación de entrevista.

En la perspectiva etnosociológica, la cuestión esencial es saber si se puede fiar uno de 
relato de vida como descripción del itinerario biográfico o, más exactamente, en quémedid se puede uno fiardeél. (Bertaux, 2005:77)

— La realidad psíquica y semántica de lo que el sujeto sabe y piensa retrospectivamente acerca de su itinerario biográfico, a partir de la totalización subjetiva de sus experiencias vividas. Se tiende a suponer, salvo que haya indicios en el sentido contrario, que el sujeto ha memorizado correctamente los acontecimientos más relevantes y su orden temporal.

- La realidad histórico-empírica de la historia realmente vivida, "lo que realmente sucedió", el itinerario biográfico conformado por las situaciones, acontecimientos y acciones que vivió el sujeto. La perspectiva etnosociológica, sin desconocer los otros dos órdenes de realidad, se enfoca en este último, con el fin de conocer los mecanismos de funcionamiento de un objeto social y las lógicas de acción en las relaciones sociales.

Para que este tipo de análisis sea válido y eventualmente generalizable, se necesitan algunas pautas y recaudos:

- Analizar varios testimonios simultáneamente, con una vocación comparativa - lo que también facilita el control de veracidad-.

- Alcanzar el punto de saturación muestral, momento en el cual la incorporación de nuevos relatos no modifica en nada sustancial la construcción progresiva de la representación del objeto sociológico de interés.

- Descubrir mecanismos genéricos como modo de aproximarse a la generalización.

La verosimilitud de las generalizaciones acerca de un modelo social depende totalmente del descubrimiento de «mecanismos genéricos», de configuraciones específicas de relaciones sociales que describen situaciones, de lógicas de acción que se ponen en práctica” (Bertaux, 2005, p. 33).

\section{Árboles genealógicos relatados}

Como complemento de los relatos de vida, los árboles genealógicos son un recurso de suma utilidad para estudiar procesos que ocurren en las familias. La metodología de "genealogías sociales comentadas y comparadas" (Bertaux, 1994) se basa en el registro de genealogías e historias familiares. Este abordaje metodológico resulta especialmente adecuado para estudiar sociedades con fuertes lazos familiares, donde estos lazos operan como canales para la movilización de recursos con el fin de colocar a los descendientes en trayectorias sociales deseables.

El término familia es relativo a un sujeto, no tiene contornos definidos. La familia ampliada de una persona está formada por varias familias nucleares. Entonces, ¿cómo se define dónde termina una familia al graficar? El grupo familiar que Bertaux considera recomendable graficar incluye, por lo menos, las dos parejas de abuelos de Ego —el sujeto entrevistado y/o foco de interés - y, a partir de ellos, todos sus hijos con sus respectivos cónyuges y descendientes. Si bien habitualmente se realizan representaciones egocéntricas en los árboles genealógicos, no debe olvidarse que cada pariente tiene sus propios árboles familiares: uno "hacia arriba", de ancestros, y otro "hacia abajo", de descendientes, que si se incluyeran representarían un tupido bosque de relaciones de parentesco, tanto de filiación como de alianza. La información que debe incluirse en la gráfica depende de los objetivos de investigación, pero por lo general se necesita consignar sexo, edad o año de nacimiento - y de muerte, de ser el caso-, lugar de nacimiento y/o de residencia actual, ocupación principal y nivel educativo. Esta información sociodemográfica básica, al presentarse en formato de árbol, permite contextualizar en familias los atributos individuales de un modo que se capta a simple vista. Así, se hace posible examinar las interacciones entre las historias individuales y descubrir regularidades, por ejemplo, comparar y relacionar trayectorias ocupacionales de grupos completos, entre hermanos, o de tíos con sobrinos, etcétera. Idealmente las genealogías deberían llegar a ser socio-centradas: en vez de un árbol genealógico individual, un rectángulo o ventana de observación de una amplia red de parentela y alianzas.

La genealogía sugiere pistas sobre cómo sucedió la historia, pero no sobre por qué sucedió de esa manera. Las historias familiares que emergen en los relatos de vida permiten esto último, ya que contribuyen a hacer visibles algunos de los procesos claves, por ejemplo, aspiraciones frustradas de los padres y luego proyectadas, una fábrica que abre o cierra en determinado lugar, etc. Mientras que la genealogía estandariza los hechos, las historias familiares proporcionan el significado de esos hechos y aportan otros nuevos, configurando cadenas causales de acciones y sucesos que, al repetirse en varios casos, permiten identificar patrones para generalizar. Las genealogías en combinación con los relatos de vida contienen información sobre varones y mujeres de al menos tres generaciones; consignan atributos individuales y grupales con una dimensión temporal continua; registran itinerarios de vida en los planos ocupacional, familiar y residencial; informan sobre los contextos locales o regionales; $y$ aprehenden significados retrospectivos de las historias.

Una vez analizados los casos de familias por separado, el paso siguiente es la comparación de familias con antecedentes similares - por ejemplo, una misma ocupación de los abuelos-enfocándose en los procesos de diferenciación de los itinerarios. El análisis comparativo intra-clases sociales - $\mathrm{O}$ al interior de grupos de otro tipo que engloben a una categoría de personas- debería permitir identificar el campo de posibilidades para un origen social dado en determinado espacio y tiempo. Luego, es posible compa- rar entre clases, examinar cuánto difieren los campos de posibilidades, qué factores de diferenciación son los principales y qué factores en común se encuentran.

Una experiencia de caso: investigación cualitativa sobre mecanismos de movilidad social en Buenos Aires

En este apartado quisiéramos hacer algunas reflexiones metodológicas a partir de una investigación sobre movilidad socia desde el abordaje cualitativo propuesto por Bertaux. El estudio se proponía describir trayectorias intergeneracionales desde distintos orígenes de clase - el origen de clase convencionalmente se determina a partir de la ocupación de los padres de una persona-. Para ello, se seleccionaron 35 casos de distintas posiciones de clase con el fin de obtener relatos de vida sobre el itinerario laboral y educativo familiar.

La guía de pautas para las entrevistas, entendida como un guion flexible, consistió en un conjunto organizado de temas y subtemas, incluyendo posibles preguntas no estandarizadas. La consigna inaugural introdujo a los entrevistados en el interés por conocer su recorrido laboral y familiar, proponiéndoles, como punto de partida del diálogo, que cuenten la historia de su familia, comenzando por abuelos o bisabuelos, hasta llegar a su propia historia personal. Se orientó la conversación procurando seguir el orden cronológico de las generaciones y de las etapas de la vida del entrevistado.

Los ejes temáticos estuvieron orientados a obtener información sobre distintas forma de capital familiar - económico, cultural social, simbólico-(Bourdieu, 1990), lo que implicó la necesidad de indagar en aspectos económicos, laborales, educativos, familiares, de sociabilidades y los referidos a aquellos "mundos sociales" específicos o ámbitos 
de existencia de los cuales los entrevistados forman parte. Interesaron especialmente en cada eje temático las cuestiones cronológicas referidas a la duración de las etapas y transformaciones vitales, los momentos cruciales de cambio, los mecanismos facilitadores u obstaculizadores para el logro de aspiraciones personales, y los modos de transmisión de recursos familiares.

Asimismo, mediantela técnica del árbol genealógico, se registraron informaciones que permitieron relacionar las oportunidades de contexto socio-histórico con las trayectorias de las distintas generaciones y con la transmisión de habilidades, ocupaciones y recursos.

Se procuró que tanto los relatos como los árboles genealógicos contengan cierta información básica para todos los entrevistados. En los árboles se consignó, como mínimo, ocupación, año de nacimiento y nivel educativo de Ego, abuelos, padres, hermanos y pareja. Para Ego, se consignó el trabajo actual o el más reciente. Para los padres y la generación anterior, el trabajo de mayor relevancia en la vida laboral activa. De acuerdo al relato de cada entrevistado se incluyeron eventualmente otros miembros de la familia, como tíos o parientes políticos, especialmente cuando tuvieron un rol relevante en la historia personal o familiar.

\section{"Hallazgos" en el trabajo de campo}

El trabajo de campo con relatos de vida permitió descubrir cómo funcionaban en la práctica, para la muestra relevada, las técnicas empleadas. En primer lugar, los árboles genealógicos de distintas familias tenían distintas estructuras. En algunos casos ello dependía de la composición familiar, pero en otros se debió a diferencias en el conocimiento y memoria familiar de los entrevistados, así como a variaciones en las pautas familiares en distintas posiciones en el espacio social. En las familias de menor capital cultural, las genealogías a veces co- menzaban en los padres del entrevistado y se extendían hasta nietos, al contrario de las genealogías de familias de mayor capital cultural, en las que siempre se recordaba a los abuelos, no pocas veces a bisabuelos, y no solía haber aun nietos o siquiera hijos.

En segundo lugar, los sujetos que aceptaron ser entrevistados se mostraron bien predispuestos a relatar sus historias de familia. La consigna inaugural de contar la historia familiar comenzando por los abuelos funcionó particularmente bien al dar lugar a que los entrevistados inmediatamente tuvieran ganas y confianza para desarrollar el relato. A menudo, las entrevistas comenzaron con un primer turno de palabra de varios minutos del entrevistado sin necesidad de que el entrevistador interrumpiese o dirigiese el relato. En los minutos iniciales de la entrevista aportaron una gran cantidad de información fáctica que debía relevarse, de varios ítems de la guía de pautas de entrevista, y buena parte de la información para los árboles, sin necesidad de que se los interrogara puntualmente por cada cuestión. Esto constituye una ventaja sobre la encuesta, al lograr que los entrevistados estén motivados para brindar información, que puedan hacerlo sin respuestas preestablecidas y confiriendo sentido a las informaciones en el marco de una narración que las unifica.

La principal desventaja que se observó en el análisis fueron las diferencias en e nivel de detalle en ciertos aspectos de los distintos relatos. Si bien los grandes ejes de entrevista fueron cubiertos en todos los casos, respecto a cuestiones específicas se encontró que para algunos entrevistados se tenía información y para otros no. Por ejemplo, cuando algún entrevistado mencionaba numerosos empleos por los que pasó, no resultaba adecuado para una entrevista realizarle una serie de preguntas estandarizadas para caracterizar cada uno de ellos, pero esto implicó que no se cuente con la misma información para todos.
Con respecto a los ingresos y al patrimonio económico, la falta de estandarización también tuvo la desventaja de la vaguedad es decir, que la información estuviese demasiado teñida por la forma de construcción de cada relato. En compensación, la riqueza de los relatos contuvo los contextos y los acontecimientos necesarios para reconstruir los aspectos económicos más sustanciales, haciendo menos gravosa la falta de precisión con respecto a algún indicador específico.

Incluso cuando las preguntas se asemejaban a las típicas de encuesta, la entrevista permitió poner de manifiesto lo que se daría por sentado y lo que escaparía a una respuesta de cuestionario. Por ejemplo, las preguntas referidas a cuál fue el primer trabajo y a qué edad, que parecen no presentar complejidad, no siempre tienen respuestas precisas que puedan situarse en un momento exacto de la historia. En una encuesta no emergerían aclaraciones significativas, como la de un entrevistado que en la infancia pasaba gran parte de su tiempo en el negocio de su padre y por lo tanto el pasaje entre juego y trabajo no fue un momento preciso.

En lo referido al capital cultural, la entrevista permitió no sólo analizar el contenido de lo dicho por el entrevistado sino además el propio lenguaje y modos de expresarse, queen conjunto permiten conocer suficiente sobre este capital en cada caso. El capital socia ha sido el de más dificultosa aprehensión. No pocas veces la información más relevante que los entrevistados proporcionaron al respecto emergía en comentarios hechos a pasar, digresiones o explicaciones secundarias en la estructura de los relatos. Por ende, la información que se obtenía difería según las formas de comunicar de cada entrevistado. Por ejemplo, si se preguntaba con el objeto de indagar en el capital social a quién podrían recurrir en caso de necesitar ayuda económica, o para encontrar un trabajo, la respuesta tendía a ser abstracta y vaga. En cambio, cuando los mismos entrevistado relataban una dificultad concreta que atravesaron, podían hacer mención a quiénes los ayudaron o a los modos en que sus relaciones sociales funcionaron en la situación específica. Entre los ejes de indagación previstos, funcionó satisfactoriamente interrogarlos por cómo consiguieron los empleos, cómo supieron acerca de una oportunidad de obtener ganancias o de la existencia de alguna organización, empresa o institución de la que han formado parte. Algunos entrevistados expresaban motu proprio estas cuestiones, lo que constituyó la situación ideal al evitar tener que inducirlos; pero los que no lo hacían solos, de todos modos, accedieron con facilidad a añadir la información que se les pedía.

\section{Acerca de cómo se hizo manejable el material relevado}

Para comenzar a explorar y compara las trayectorias, se elaboraron diagrama que permitiesen disponer la información de distintas maneras. Entre las exploraciones, se elaboró un esquema para representar el espacio social en sus dimensiones económica y cultural (Diagrama 1). Se usó la información cualitativa para clasificar a cada caso, de manera aproximada, en un nivel alto, medio o bajo de capital económico y de capital cultural. Cada caso se clasificó a partir de los indicadores disponibles en los relatos: la ocupación y el nivel educativo fueron los más relevantes - no los únicosporque se contaba con información para todos los casos. Por ejemplo, algunos entrevistados sin secundario completo fueron clasificados en capital cultural bajo y otros en capital cultural medio, según sus prácticas y consumos culturales. Del mismo modo el nivel de capital económico se definió primordialmente a partir de la información ocupacional — disponible para todos los casos-, pero también se usó como criterio suplementario la información disponible sobre propiedades e ingresos. 


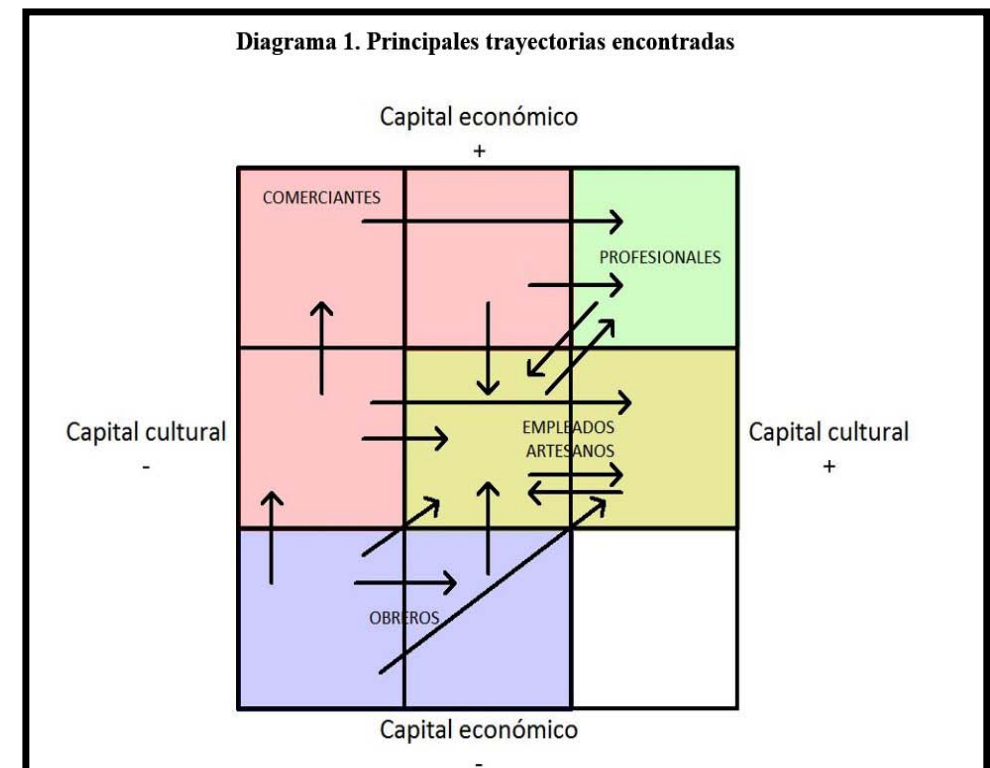

como obreros, comerciantes, profesionales, empleados y artesanos. Para los casos de mujeres se observó que predominan las flechas puntando hacia la derecha, que indica que el rumbo de su trayectorias tiende hacia el polo del mayor capital cultural.

El diagrama presenta las limitaciones de la estandarización, que es precisamente lo que se critica a los enfoques cuantitativos de la movilidad

En el diagrama, el comienzo de cada flecha representa la posición de clase de origen - por lo general, padres del entrevistado-y el final de la flecha, la posición de clase actual del entrevistado. Por ejemplo, si la generación de los padres tuvo alto capital económico y bajo capital cultural la posicionamos en el cuadrante superior izquierdo. Si la generación del entrevistado mantuvo el alto capital económico y además alcanzó un al to capital cultural, la flecha va desde la izquierda hacia la derecha -indicando el movimiento hacia el polo de capital cultural - dentro de la franja superior - porque mantuvo el al to capital económico-.

Las flechas pueden ser ascendentes, descendentes o volver al origen. Las más largas indican una trayectoria de mayor distancia social. Las horizontales y verticales indican cambio en una dimensión y permanencia en la otra. Las oblicuas, cambio de posición en ambos ejes.

Una vez elaborado el diagrama, se encontró que alounos cuadrantes tendían a coincidir con grandes categorías ocupacionales, tales bueno viste que uno se tenía que anotar, yo me había quedado sin trabajo, bueno, me vengo a anotar acáy justo necesitaban una persona que tenga experiencia en computación, en trabajos administrativos y quése yo. Y bueno empecé a trabajar acá en el CGP, cuatro horas, por una contraprestación, o sea, me pagaban 150 pesos por mes, y yo hacía la contraprestación acá todos los días cuatro horas, y a Martín lo mandaba al jardin. Ya tenía ahi dos años Martin. Y bueno, ya desde ese entonces me quedé acá, después fui contratada y después planta permanente.

Irma, empleada administrativa.

La condensación de una serie de eventos biográficos en fragmentos relativamente breves de relato permite concatenarlos y comprender cómo interactuaron. En la cita precedente, por ejemplo, hay información sobre: el momento histórico de Argentina en el que se desarrollan los eventos, el embarazo como acontecimiento significativo de viraje en la biografía, la situación económica y laboral familiar, la existencia de una política estatal orientada a las personas desempleadas que era conocida y percibida como accesible por la entrevistada, algunas competencias laborales obtenidas en la experiencia de trabajos anteriores, la transición de beneficiaria de un programa social a trabajadora estable, la duración de la jornada, la remuneración del trabajo y la compatibilización de ese trabajo con las tareas de cuidado del hijo, facilitada por la jornada reducida —en principio, porque las tareas no eran definidas oficialmente como trabajo sino como contraprestación de un beneficio-. A su vez, la sincronización de estos eventos en el relato permite comprender el sentido de cada uno de ellos en relación a todos los demás. Lo que podría ser una información entre otras, como la obtención de un subsidio, cobra un sig nificado decisivo para la comprensión de la trayectoria cuando los demás sucesos le otorgan tensión narrativa al relato y ponen de manifiesto su centralidad.

Por otra parte, en este microrrelato aparece la conexión entre el tiempo presente en que se sitúa la narración, los sucesos pasado -como las experiencias laborales previas, desarrolladas anteriormente en la entrevista-y os futuros. Asimismo, queda de manifiesto cómo, en vez de una promoción mediante la carrera laboral, el "ascenso" ocurrió a través de la formalización de su empleo o la salida de la precarización, que asume en este relato un papel análogo al que en otros ocupa la descripción del logro ocupacional. Las cuatro veces que menciona a su hijo sugieren que el cuidado era parte fundamental del asunto que describe, pues un empleo de jornada completa no le habría resultado conveniente. Se sobreentiende que el hijo asistía al jardín de infantes en el horario en que ella concurría a trabajar, y que de ese modo se resolvieron las circunstancias entrelazadas que expuso.

La riqueza de significados contenidos en os relatos permite efectuar interpretaciones suficientemente informadas y significativas, valiéndose de las propias síntesis que ofrecen los entrevistados sobre sus prácticas. La clase, el género, la carrera laboral, las tareas de cuidado, y cualesquiera otras dimensiones bajo análisis, se presentan unificados en las experiencias concretas.

- Situaciones poco habituales o no previstas

Cuando se trabaja con la técnica de encuesta ciertamente se pueden incluir preguntas de lo más variadas en los cuestionarios para capta todo tipo de informaciones. Sin embargo, a menudo se necesita agregar nuevas preguntas para detectar ciertos matices. Estas preguntas pueden resultar superfluas o redundantes para la mayoría de los encuestados, pero se necesita incluirlas en el cuestionario para captar los casos específicos para los cuales fueron pensadas. Anivel local, por ejemplo 
en el Censo Nacional de 1980 se preguntaba: ¿Qué hizo la semana pasada? ¿Trabajó? ¿No trabajó pero tenía empleo? Al evaluar los censos se detectó el subregistro de la actividad económica de las mujeres, entre otras categorías poblacionales, porque algunas tendían a no considerar trabajo ciertas actividades que, si bien estaban orientadas al mercado, eran irregulares o de pocas horas. Se decidió entonces preguntar: Durante la semana pasada, jtrabajó aunque sea por pocas horas? ¿Hizo algo en su casa para afuera o ayudó a alguien en su negocio, chacra o trabajo? ¿Estuvo de licencia por enfermedad, vacaciones, etc.?

Ciertas preguntas, por más sencillas que sean, pueden comportar significado distintos según diversas circunstancias (Cohen y Gómez Rojas, 2014, p.15)

Si bien en ocasiones unas preguntas pueden ser sustituidas por otras más precisas, lo más habitual es que para ampliar la sensibilidad del instrumento de registro se requiera añadir nuevas preguntas, lo que torna más engorroso el cuestionario. Además, no siempre pueden contemplarse todos los aspectos en que los fenómenos relevantes pueden desbordar la capacidad de registro del instrumento. En este sentido, una de las ventajas de los relatos de vida es que permiten toparse con informaciones no previstas.

Una de estas situaciones se presentó con los individuos que tuvieron simultáneamente padres biológicos y adoptivos. El padre de una entrevistada (Fabiana) había sido criado por una vecina, porque su madre al enviudar no estaba en condiciones de mantener a tantos hijos. Eso implicó que la trayectoria posterior del niño siguiera las pautas de la familia adoptiva, pese a que no perdió contacto con su madre biológica. En otros dos casos, que se presentan a continuación, las entrevistadas tuvieron situaciones similares de "doble origen", cada una con sus particularidades.

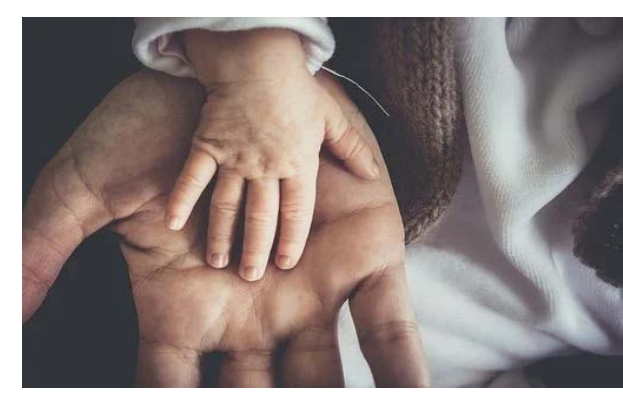

\section{Caso A. Miriam}

Miriam fue criada en el hogar de sus padres biológicos, que eran medianos propietarios rurales en Santiago del Estero. Cuando tenía seis años, en 1972, padeció enfermedades respiratorias y su familia decidió llevarla a Buenos Aires para que sea tratada. La familia no se trasladó, sino que dejó a Miriam al cuidado de una de las hermanas de la madre que vivia en Buenos Aires. La enfermedadse prolongó algunos años, se sucedieron varias internaciones y con el paso del tiempo el hogar de sus tíos pasó a ser su familia adoptiva. Los padres biológicos, en palabras de Miriam "no la reclamaron". Los tios, que no podian tener hijos pese a que lo deseaban, la criaron desde entonces como a su hija. La situación económica de ambos hogares difería bastante, por lo cual la mudanza implicó un cambio de origen de clase, desde una familia rural de medianos propietarios a una familia obrera urbana, que incluso alquilaba su vivienda Este cambio de origen social tuvo consecuencias en la trayectoria de Miriam. La situación económica era precaria, porque el tío enfermó de encefalitis y solamente la tía proveía ingresos al hogar con su trabajo de modista durante toda la infancia y adolescencia de Miriam. En cuanto al capital cultural, parece haberse impuesto la herencia de la familia biológica. Miriam fue docente al igual que sus hermanas. Recientemente logró concluir una carrera universitaria, algo que habría querido realizar de joven pero que fue obstaculizado por la frágil situación económica.

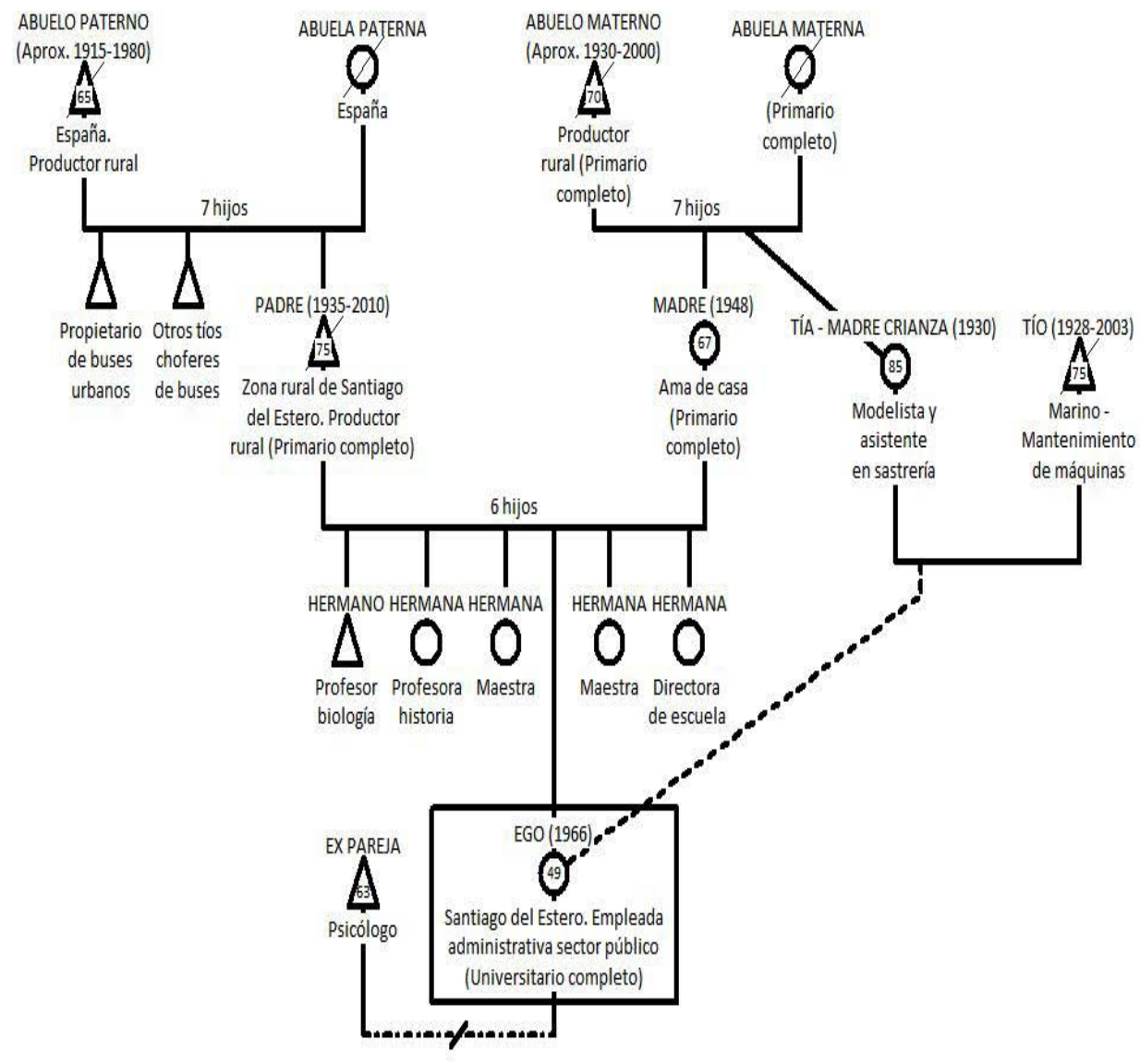

\section{Caso B. Marisa}

Marisa también ha tenido un doble origen, pero las circunstancias fueron distintas. $L a$ madre biológica era una empleada doméstica analfabeta, que tuvo a Marisa con el hijo de suspatrones. Estos últimos no creyeron quesu bijo fuera el padre de la beba y la despidieron. Luego trabajó algún tiempo en otra casa de familia, la de una martillera pública inglesa en pareja con un músico. Alpoco tiempo se fue y abandonó a su hija con ellos, que la criaron.

El capital económico y cultural de la familia de crianza era inconmensurablemente superior al de su madre biológica. La crianza con la familia adoptiva le ha significado a Marisa una trayectoria con oportunidades propias de aquella posición de clase, con la salvedad de que no le ha correspondido ninguna parte del patrimonio en la sucesión de sus padres adoptivos. De grande conoció a sus padres biológicos y mantiene contacto con ellos. 


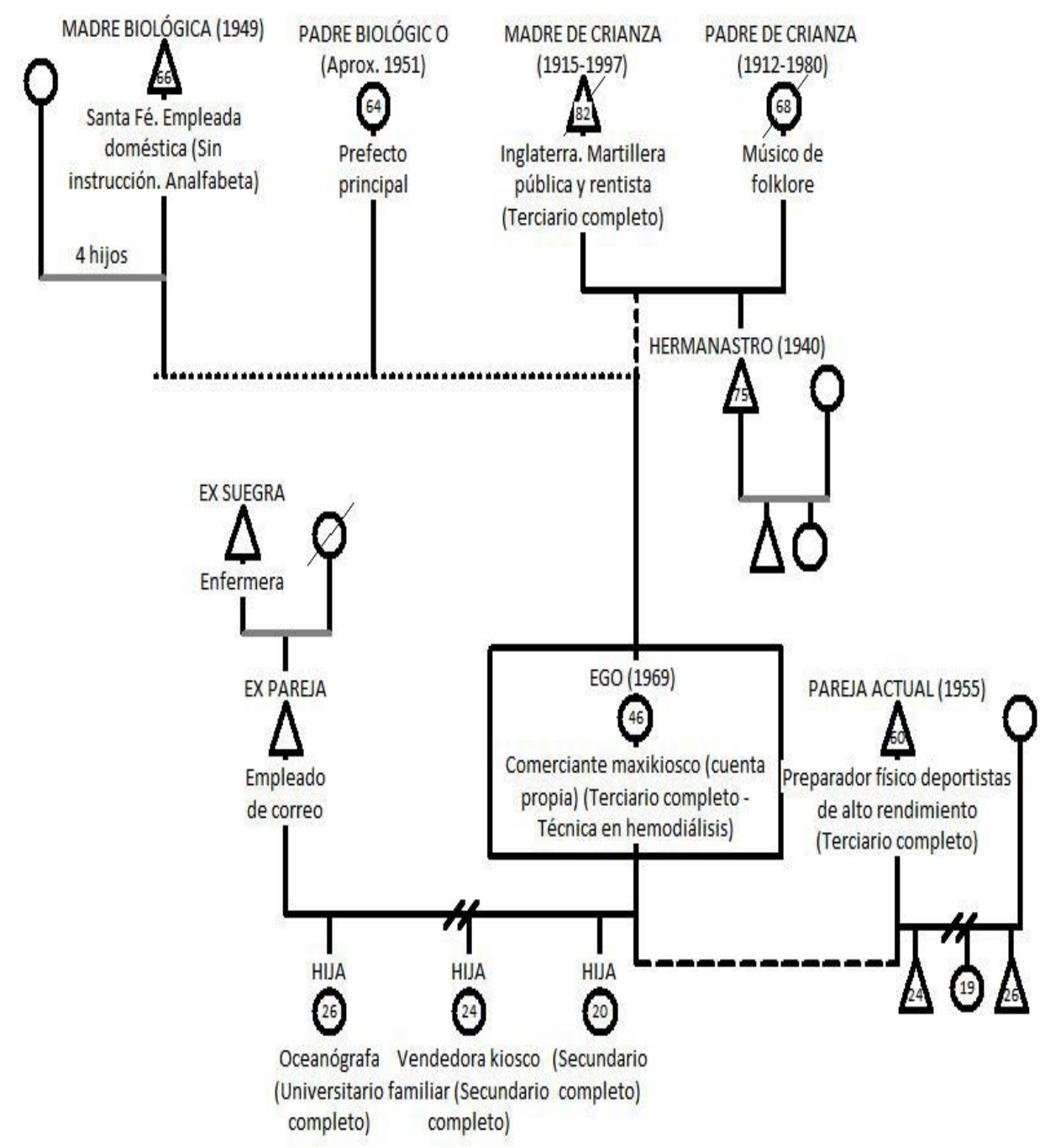

Como puede advertirse, en estos casos en los que hubo un doble origen familia tiende a haber herencias de ambas familias y la trayectoria se define a partir de la combinación de legados específicos en cada caso. La familia biológica y la de crianza pueden coexistir con distintos grados de presencia en cada etapa de la vida. Las adopciones de hecho - en los tres casos- tienen efectos a muy largo plazo en las herencias económicas tras la muerte de unos y otros padres, privan-

do a los hijos del derecho a tomar parte en la sucesión (Marisa) o bien permitiéndoles cobrar en el futuro una herencia de padres que estuvieron ausentes (Miriam). El capital cultural y social heredado de una y otra familia dependen de la edad en la que tuvo lugar la adopción de hecho, pero también de las idiosincrasias familiares.

Este ejemplo, hallado en el conjunto de relatos analizados, se puede añadir a muchos otros de distinto tipo señalados por Bertaux y Thompson (2007). Existen limitaciones intrínsecas a la investigación cuantitativa -así como las respectivas de las investigaciones cualitativas - que desaconsejan que sea la única vía legítima para estudiar las distinta aristas de la movilidad social. Las situaciones atípicas que se desconocen al estandarizar pueden tener un potencial heurístico que se está desaprovechando. A partir del ejemplo precedente, cabe preguntarse si los individuos que tuvieron por distintas razones un doble origen de clase no resultarían especialmente reveladores para la comparación, por ejemplo, en investigaciones cualitativas de inducción analítica o inspiradas en los clásicos procedimientos experimentales de combinación de concordancias y diferencias formulados por John Stuart Mill. locales

- Encadenamientos de causalidade

Los procesos de movilidad social involucran una serie de factores que han sido identificados y estudiados, por ejemplo, en los enfoques cuantitativos del logro de status, donde se ponen a prueba distintos modelos para evaluar cuáles combinacione de variables ofrecen mayor poder explicativo (Blau y Duncan, 1967). Los relatos de vida pueden integrarse con investigaciones cuantitativas de este tipo, sin circunscribirse a un rol necesariamente subsidiario. Tanto para generar teoría como para visualizar cómo se expresan las regularidades estadísticas en casos concretos, los relatos de vida ofrecen información rica y sugieren interpretaciones. Al permitir reconstruir la secuencia de ciertos acontecimientos en las biografías, se comprenden más adecuadamente los procesos de movilidad. El siguiente fragmento de relato puede ilustrar este punto.

Saqué un crédito en el Banco C., un crédito que le daban a los choferes de taxi. Yo siendo chofer lo pude sacary compré coche y licencia. Pero pagaba un montón, como nueve lucas por mes de crédito nomás. Aparte tenía los gastos del coche. Encima el coche que compré no le pude poner gas, lo tenía a nafta. Por el problema que tuvo mi señora [está bajo prisión domiciliaria] no pude trabajar las horas que tenia que trabajar. Empecé a atrasarme en los pagos del crédito y lo terminéperdiendo. $Y$ quedé endeudado con el banco, todavía estoy endeudado con el tema de la licencia (...). El taxi yo lo perdi a los meses de que ella tuvo el problema... a los diez meses. Pero ese problema fue lo que me complicó toda la vida, porque ella hacía todo en la casa, buscaba a los chicos, los traía, se ocupaba de ellos. Yo solamente me ocupaba de laburar.

Facundo, repositor en supermercado.

La desigual distribución de riesgos según la posición social, especialmente de acuerdo al capital económico y cultural permite comprender bajo qué condiciones se activan las espirales de desventaja (Mora y de Oliveira, 2014), como la de la familia de Facundo. No son los factores en abstracto como "bajo nivel educativo" lo que originan estos procesos, sino que lo hacen a través de las mediaciones y formas particulares que adoptan en cada proceso concreto. La categorización de los factores intervinientes se ajusta mejor a los fenómenos si es elaborada a partir de los datos que si se asume a priori o se da por sentada. La secuencia en que se desenvuelven los acontecimientos puede ser decisiva en su resultados, porque si tuvieran lugar en otro orden, no se producirían las interacciones y los efectos serían distintos.

\section{Conclusiones}

Los relatos de vida permiten aprehender algunos aspectos de fenómenos estructurales, por ejemplo, cuestiones de movilidad social, que tienden a permanecer opacos en los abordajes cuantitativos. A menudo fragmentos incluso breves de relato contienen una gran riqueza informativa y de significados en torno a hechos biográficos. 
Pero no sólo se adicionan informaciones, sino que se repone la unidad de los procesos, las concatenaciones de eventos y el sentido de cada evento en relación a todos los otros. Las situaciones poco habituales o no previstas, que requerirían para ser captadas añadir más y más preguntas en un cuestionario, emergen en cambio con facilidad en los relatos. Y no por atípicas dichas circunstancias resultan necesariamente poco significativas, por ejemplo, en la investigación sobre trayectorias de clase social se encontró mediante los relatos de vida y los árboles genealógicos que los niños que tuvieron por distintas razones dos hogares de distintas posiciones de clase en su infancia, combinaban ulteriormente herencias de ambas familias en sus trayectorias de clase.

La comparación entre relatos de personas o familias que parten de similares condiciones permite descubrir pautas o patrones. Ala vez, las historias contenidas en los relatos facilitan la identificación de los mecanismos que originan diversificaciones en las trayectorias y procesos estudiados. Los árboles genealógicos, cuando contienen la información adecuada para la investigación, resultan reveladores de significativas regularidades familiares, por ejemplo, ocupaciones que se transmitieron de tías a sobrinas, que probablemente se pasarían desapercibidas en una investigación mediante encuesta. Así, la combinación de relatos de vida y árboles genealógicos abre posibilidades para explorar otros carices de los fenómenos estructurales, tradicionalmente abordados mediante estudios por encuesta.
-Bertaux, D. (1999). El enfoque biográfico: su validez metodológica, sus potencialidades. En Proposiciones 29. Traducido de L'approche biographique: Sa validité méthodologique, ses potentialités, publicado en Cabiers lnternationaux de Sociologie, Vol. LXIX, París, 1980, pp. 197-225.

- Bertaux, D. (2005). Los relatos de vida. Perspectiva etnosociológica. Barcelona: Edicions Bellaterra.

-Bertaux, D. y Thompson, P. (2007) (eds.) Pathways to social class. A qualitative approach to socialmobility. New Brunswick: Transaction Publishers.

-Blau, P.y Duncan, O. (1967). The American Occupational Structure. Nueva York: Wiley.

-Bourdieu, P. (1990). Espacio social y génesis de las clases. Sociología y Cultura. México: Grijalbo.

—Cohen, N.y Gómez Rojas, G. (2014). Esa cosa llamada datos. Revista Latinoamericana de Metodología de la Investigación Social, $\mathrm{N}^{\circ}$ 8. Año 4, pp. 10-18.

—Kornblit, A. L. (2007). Historias y relatos de vida: una herramienta clave en metodologías cualitativas. En Metodologías Cualitativas en Ciencias Sociales. Buenos Aires: Biblos.

-Scribano, A. (2008). El proceso de investigación social cualitativo. Buenos Aires: Prometeo.

\section{Bibliografia}

-Bertaux, D. (1994). Genealogías sociales comentadas y comparadas. Una propuesta metodológica. Estudios sobre la cultura contemporánea, Vol. VI, No16-17, México: Universidad de Colima, pp. 333-349. 